\title{
Isolated carotid artery fibromuscular dysplasia unifocal type diagnosed in the postpartum period
}

\author{
Karolina Woziwodzka ${ }^{1}$, Katarzyna Krzanowska ${ }^{1}$, Małgorzata Szafirska ${ }^{2}$, Marcin Krzanowski ${ }^{1}$, \\ Artur Dziewierz ${ }^{3}$, Władysław Sułowicz ${ }^{1}$ \\ ${ }^{1}$ Department of Nephrology, Jagiellonian University Medical College, Krakow, Poland \\ ${ }^{2}$ Department of Radiology, Jagiellonian University Medical College, Krakow, Poland \\ ${ }^{3} 2^{\text {nd }}$ Department of Cardiology, Jagiellonian University Medical College, Krakow, Poland
}

Adv Interv Cardiol 2018; 14, 2 (52): 214-215

DOI: https://doi.org/10.5114/aic.2018.76421

Fibromuscular dysplasia (FMD) is an idiopathic noninflammatory and nonarteriosclerotic segmental disorder of almost every arterial bed, leading to arterial sclerosis, occlusion, aneurysm, or dissection. It occurs most frequently in renal (80\%), extracranial carotid and vertebral arteries (25-30\%). Isolated intracranial FMD develops in $8.3 \%$ of patients. Carotid artery FMD is typically located bilaterally, in the middle and distal parts of the carotid artery [1, 2]. There are two characteristic angiographic types of FMD: multifocal with a typical string-of-beads appearance; and unifocal, characterized by stenosis [1]. Patients with carotid or vertebral artery FMD present with numerous nonspecific symptoms. However, specific neurological signs, such as transient ischemic attack, Horner syndrome, cranial nerve palsies, cerebral infarction, amaurosis fugax, and subarachnoid hemorrhage, may also be observed [3].

A 39-year-old woman with Graves disease, after radioisotope therapy, and suffering from polycystic ovary syndrome, was admitted to the hospital due to paraparesis reported on the third day postpartum. Two years earlier she suddenly developed transient right Horner syndrome, alteration in the bitter taste (a symptom of dysgeusia), and right mandible and ear pain. Duplex ultrasound (DUS) performed 10 days after the neurological episode revealed occlusion of the right internal carotid artery (RICA) with very limited blood flow, similarly to computed tomography angiography (CT angiography). No tear or blood accumulation was revealed.

At the beginning of her third pregnancy, she experienced periodic episodes of paresthesia of her right upper and lower extremities. Approximately during the $18^{\text {th }}$ week of pregnancy, the symptoms of paresthesia in her left leg and arm became more pronounced. Finally, due to the loss of sensation on the left side of her body and propriocep- tion, a caesarean section was performed in the $30^{\text {th }}$ week of pregnancy. An unexpected increase in blood pressure (200/110 $\mathrm{mm} \mathrm{Hg}$ ) following the administration of angiotensin-converting-enzyme inhibitors was observed.

During hospitalization, all neurological symptoms gradually resolved. Another DUS examination showed a narrow RICA with an irregular lumen. No other changes constricting the lumen were detected. Computed tomography angiography revealed patent (1-2 $\mathrm{mm}$ ) tortuous course of the right extracranial carotid artery (Figures $1 \mathrm{~A}, \mathrm{~B}$ ), patent $(1-1.8 \mathrm{~mm})$ intracranial carotid artery with dilation to $2.5 \mathrm{~mm}$ in its cavernous part (Figure $1 \mathrm{C}$ ), and well-developed compensatory circulation from the circle of Willis via the anterior and posterior communicating arteries (Figure $1 \mathrm{D}$ ). The unifocal type of FMD was diagnosed. No changes in the vertebral or renal arteries and no other abnormalities in the circle of Willis were detected. The patient's clinical condition significantly improved with resolution of neurological symptoms, and blood pressure was well controlled with amlodipine (110/80 mm Hg on average). Because of the compensatory circulation and the lack of neurological symptoms, no balloon angioplasty was performed.

Interestingly, in this case, spontaneous carotid artery dissection (SCAD) may be superimposed on FMD, which has been observed in up to $90 \%$ of SCAD patients. Unfortunately, even angiography may be inadequate to differentiate the cause of the artery narrowing [4]. Perhaps the pregnancy was a trigger of the second episode of neurological symptoms.

\section{Acknowledgments}

Financial support was provided by statutory grant K/ZDS/000597 from the Jagiellonian University Medical College to KK.

\section{Corresponding author:}

Katarzyna Krzanowska MD, PhD, Department of Nephrology, Jagiellonian University Medical College, 15 C Kopernika, $31-501$ Krakow, Poland, phone: +48 606133 133, e-mail: kasiajanda@op.pl

Received: 25.02.2018, accepted: 15.04.2018. 

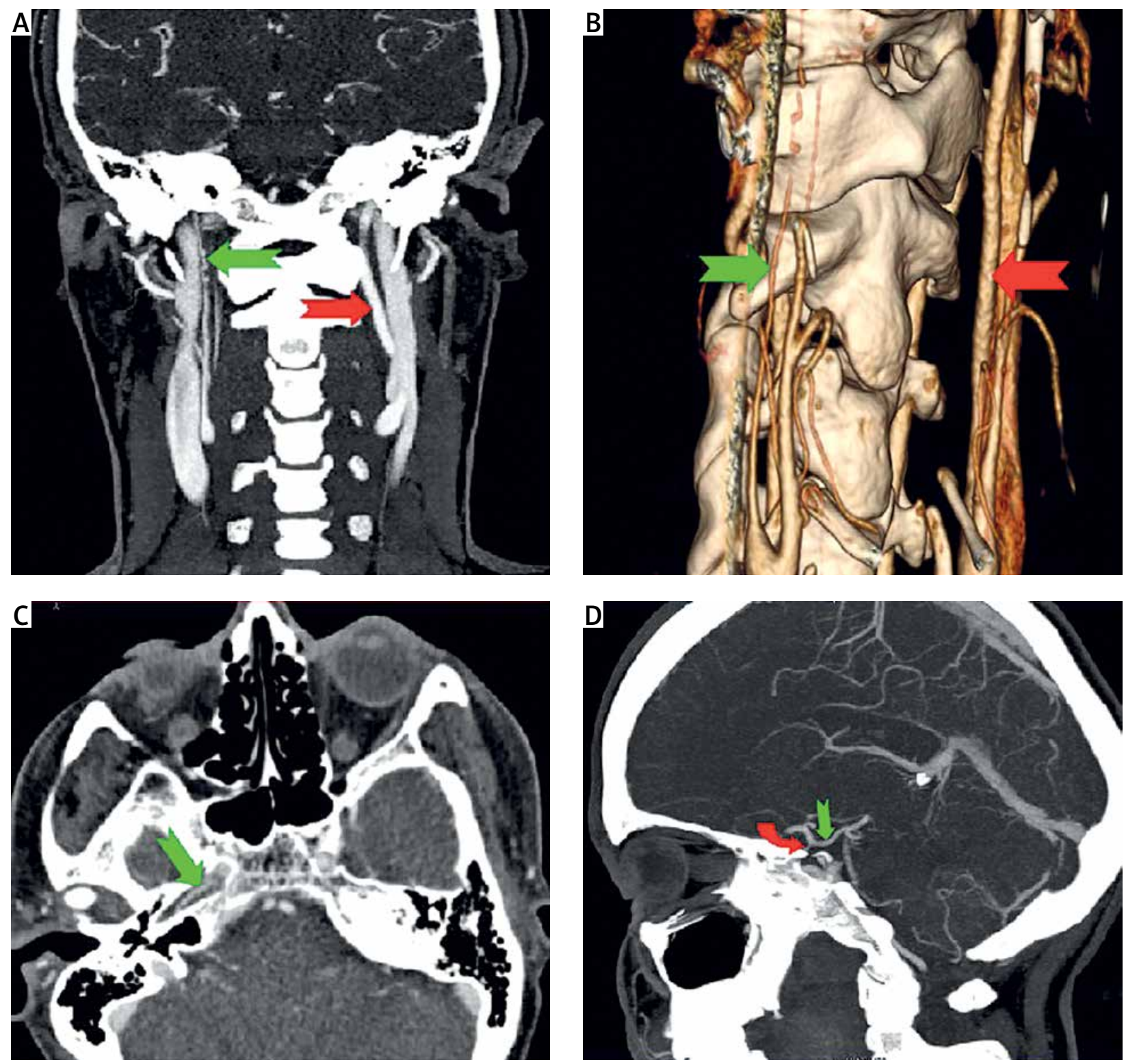

Figure 1. A - Coronal plane of the maximum intensity projection reconstruction of a computed tomography angiography scan: stenotic and winding right extracranial internal carotid artery (green arrow). The normal size of left internal carotid artery (red arrow). B - 3D reconstruction of angio-CT examination: stenotic right extracranial internal carotid artery (green arrow). The normal size of left internal carotid artery (red arrow). C - Axial plane of the maximum intensity projection reconstruction of a computed tomography angiography scan: a stenotic right extracranial internal carotid artery (green arrow). D - Sagittal plane of the maximum intensity projection reconstruction of a computed tomography angiography scan: a stenotic intracranial internal carotid artery (red arrow) and compensatory circulation from the circle of Willis via an expanded right posterior communicating artery (green arrow)

\section{Conflict of interest}

The authors declare no conflict of interest.

\section{References}

1. Persu A, Giavarini A, Touzé E, et al. European consensus on the diagnosis and management of fibromuscular dysplasia. J Hypertens 2014; 32: 1367-78.

2. Kaszuba AM, Prejbisz A, Kądziela J, et al. Forty-two-year-old female patient with resistant hypertension, bilateral renal fibro- muscular dysplasia and intracranial aneurysm. Adv Interv Cardiol 2016; 12: 386-8.

3. Olin JW, Froehlich J, Gu X, et al. The United States Registry for fibromuscular dysplasia: results in the first 447 patients. Circulation 2012; 125: 3182-90.

4. Saw J, Sedlak T, Ganesh SK, et al. Spontaneous coronary artery dissection (SACD). Circulation 2015; 131: e3-5. 